ROM2F /2004/19

hep-th/0407134

\title{
D-Brane Dynamics in Dp-Brane Background
}

\author{
Kamal L. Panigrahi* ${ }^{\dagger}$ \\ Dipartimento di Fisica, Universita' di Roma "Tor Vergata" \\ INFN, Sezione di Roma "Tor Vergata", Via della Ricerca Scientifica 1 \\ 00133 Roma, Italy
}

\begin{abstract}
By using Dirac-Born-Infeld action we study the real time dynamics of $D$-branes in the vicinity of a stack of $D p$-branes where the role of the tachyon of the open string models is played by the radial mode on the $D$-branes. We examine the behaviour of the tachyon potential and study the hamiltonian formulation and classical solutions of such systems. We also study the homogeneous solutions of the classical equations of motion in these cases.
\end{abstract}

*e-mail: Kamal.Panigrahi@roma2.infn.it

$\dagger$ INFN fellow 


\section{Introduction}

In the study of tachyon dynamics, in open string theories, construction of various time dependent classical solutions has been an important area of research in the recent past. These solutions represent the decay of an unstable D-brane or a braneantibrane pair as the tachyon rolls towards the minimum of the potential[1]. The open string field theory on these systems have a natural time dependence and they are shown to be spatially homogeneous classical solutions of arbitrary low energy density [1, 2, 3, 4, 5, 6, 17, 8, 9, 10. These solutions, in general, are constructed by perturbing the boundary conformal field theory that describes the D-brane by an exact marginal deformation. The real time tachyon dynamics shows that effective action for the Dirac-Born-Infeld type [11, 12, 13, 14] captures many aspects of rolling tachyon solutions of the full string theory. The rolling tachyon solutions predicts that at late times tachyon condensation leads to a tachyon matter state which has the equation of state similar to that of a pressure-less fluid, and also there is no on-shell open string modes present. The open string picture of this process of time evolution leads to a new kind of open-closed string duality [15, 16]. In [5], it has been shown that in the theory of Born-Infeld coupled to gravity, at late time, the tachyon field $T$ appearing in the action could serve as the definition of time for the canonical gravity problems. So the speculation is that the tachyon field $T$ could also lead to the identification of an intrinsic time variable in the full string theory. A lot of work has also been dedicated in the analysis and the relevance of tachyon condensation in cosmological applications [17, 18, 19, 20, 21, 22, 23, 24, 25].

However in a recent paper by Kutasov [26] this problem has been analyzed by using the Dirac-Born-Infeld (DBI) action of a $D p$-brane in the vicinity of $N$ number of coincident NS5-branes. Though the $D$-branes are stable but in the presence of NS5branes they become unstable, as they break all space time supersymmetries. So it is probably natural to think of this process as an example of the tachyon condensation and study the closely related time evolution process of tachyon and the nature of the time dependent solutions. These has been analyzed beautifully in [26] where the role of tachyon in this whole process is played by the radial mode on the $D p$-brane. Among the other things, it was also argued and shown that the pressure goes to zero at late times like $\exp (-\alpha t)$, where $\alpha$ depends on the number of five branes and on the angular momentum of the $D p$-brane. NS5-brane background is special in the following sense. First, there is no background RR flux (hence the absence of WZ terms in the DBI action) and the harmonic function that describes the NS5-brane

supergravity solution, i.e. $H=1+\frac{N l_{s}^{2}}{R^{2}}$, makes the form of the tachyon potential grows exponentially as $R \rightarrow 0$ (where $R$ denotes the radial mode along the transverse directions of the NS5-branes). Second, the radial DBI action interpolates smoothly 
between the standard gravitational interaction between the $D$-brane and NS5-branes at large distances and a "radion matter" phase at a short distance between them [26]. This particular feature is absent in all the other $D p$-branes. Only in the S-dual picture of the above, in the region $N g_{s} \gg 1$, one could examine these effects. In all other cases, as has been shown [27, 28] the potential behaves power like and in some cases (e.g. a $D 0$-brane moving in the vicinity of $D 6$-branes, and when the probe brane and the background are of same dimension and parallel to each other etc.) one needs to include the WZ term in the full effective action. We, however, will not discuss those cases in this article. Also when there are more that one probe brane then one needs to include the Myers type of effects [29]. But still analyzing carefully the classical dynamics of branes in $D p$-brane backgrounds, and studying the time evolution process, one learns more about the nature of the time dependent solutions in string theory.

In view of the recent developments in understanding better the classical time dependent solutions, in this paper, we study the problem of the dynamics of $D$-brane near a stack of $D p$-branes by mapping it to that of a tachyon effective action of open string models and study some of its consequences. The rest of the paper is organized as follows. In section- 2 we study the effective action, the effective potential and the hamiltonian formulation for the system of a single probe brane in the background of a stack of static and parallel branes. In section-3, we study the homogeneous solutions of the equations of motion, and compute some interesting quantities like the stress tensors. In section-4, we turn our attention to somewhat interesting and closely related case of brane dynamics in the background of $D 5$-branes and examine the tachyon effective potential. Finally, in section- 5 we conclude with some discussion.

\section{Effective action and Hamiltonian Formulation}

In this section, we analyze the motion of a $D p^{\prime}$-brane in the background generated by a stack of coincident and static $D p$-branes, by using the DBI action. ${ }^{1}$ The metric, the dilaton $(\phi)$ and the R-R field $(C)$ for a system of $N$ coincident $D p$-branes is given by:

$$
\begin{aligned}
& g_{\alpha \beta}=H_{p}^{-\frac{1}{2}} \eta_{\alpha \beta}, \quad g_{m n}=H_{p}^{\frac{1}{2}} \delta_{m n}, \quad(\alpha, \beta=0, . ., p ; m, n=p+1, \ldots, 9), \\
& e^{2 \phi}=H_{p}^{\frac{3-p}{2}}, \quad C_{0 \ldots p}=H_{p}^{-1}, \quad H_{p}=1+\frac{N g_{s} l_{s}^{7-p}}{r^{7-p}} .
\end{aligned}
$$

\footnotetext{
${ }^{1}$ We consider the branes to be static, and ignore the massive closed-string modes. Hence the low-energy expansion is valid (neglecting $\alpha^{\prime}$ corrections). These modes become relevant at $r<<l_{s}$. But here we assume: $l_{s}^{7-p}<<r^{7-p}<<N g_{s} l_{s}^{7-p}[26$. The DBI analysis is perfectly valid in this domain and we can study the dynamics of branes in terms of effective action.
} 
where $H_{p}$ is the harmonic function of $N D p$-branes satisfying the Green function equation in the transverse space. In the subsequent analysis we assume (1) $p>p^{\prime}$, (2) there is no magnetic flux on the branes, and (3) there is a single probe brane at a time. Since $p \neq p^{\prime}$ and there is no worldvolume gauge field on the probe brane, the $\mathrm{R}-\mathrm{R}$ fields don't affect the movements of the branes and they are only affected by the gravity and by the dilaton.

The effective action on the world volume of $D p^{\prime}$-brane is governed by the DBI action:

$$
S_{p^{\prime}}=-T_{p^{\prime}} \int d^{p^{\prime}+1} \xi e^{-\left(\phi-\phi_{0}\right)} \sqrt{-\operatorname{det}\left(G_{a b}+B_{a b}\right)} .
$$

Where $G_{a b}$ and $B_{a b}$ are the induced metric and the B-field, respectively, on the world volume of the $D p^{\prime}$-brane:

$$
\begin{aligned}
G_{a b} & =\frac{\partial X^{\mu}}{\partial \xi^{a}} \frac{\partial X^{\nu}}{\partial \xi^{b}} g_{\mu \nu} \\
B_{a b} & =\frac{\partial X^{\mu}}{\partial \xi^{a}} \frac{\partial X^{\nu}}{\partial \xi^{b}} B_{\mu \nu}
\end{aligned}
$$

where $\mu$ and $\nu$ runs over whole ten dimensional space time. The worldvolume coordinates of $D p^{\prime}$-brane are leveled by $\xi^{a}\left(a=0, \ldots, p^{\prime}\right)$, and we set (by reparametrization invariance on the world-volume of the $D p^{\prime}$-brane) $\xi^{a}=x^{a}$. The position of the Dbrane in the transverse direction gives rise to the scalars on its world-volume. We restrict ourselves to the purely radial fluctuation along the common $(9-p)$ transverse space $\left(R=\sqrt{X^{m} X_{m}\left(\xi_{a}\right)}\right)$. The induced metric on the worldvolume of the $D p^{\prime}$ brane is given by:

$$
G_{a b}=H_{p}^{-\frac{1}{2}} \eta_{a b}+H_{p}^{\frac{1}{2}} \partial_{a} R \partial_{b} R .
$$

So the DBI action of the $D p^{\prime}$-brane in the background generated by $N D p$-branes is given by:

$$
\mathcal{S}_{p^{\prime}}=-\tau_{p^{\prime}} \int d^{p^{\prime}+1} x H_{p}^{\frac{p-p^{\prime}-4}{4}} \sqrt{1+H_{p} \partial_{a} R \partial^{a} R} .
$$

The form of the above action looks somewhat similar to the DBI action of the tachyon field in the open string models, which is:

$$
\mathcal{S}_{\text {tach }}=-\int d^{p^{\prime}+1} x V(T) \sqrt{1+\partial_{a} T \partial^{a} T}
$$

Comparing the above two actions, we can define a tachyon field $T$ by the following relation:

$$
\frac{d T}{d R}=\sqrt{H_{p}(R)}=\sqrt{1+\frac{N g_{s} l_{s}^{7-p}}{R^{7-p}}}
$$


In terms of this field the "tachyon potential" in (2.6) is given by:

$$
V(T)=\tau_{p^{\prime}}\left[H_{p}(R(T))\right]^{\frac{p-p^{\prime}-4}{4}}
$$

Before solving (2.7), few remarks regarding the structure of the "tachyon potential" are in order now. One notices that for $p-p^{\prime}=4$, the above potential is equal to the tension of the probe brane. The would be tachyon mode is instead a massless scalar and the static system is supersymmetric as it preserves $1 / 4$ of the space time supersymmetry (e.g. D1-D5 system). This supersymmetry is reflected in the absence of a potential of interaction between the branes when they lie parallel to each other and are static. When $p-p^{\prime}<4$ the potential is attractive at short distances and therefore can form bound state (e.g. non-threshold bound of D1-D3 branes). When $p-p^{\prime}>4$, the effective potential is repulsive and the lowest open-string mode is massive at short distance. Many interesting facts regarding the behaviour of the effective potential in brane motions and their classical orbits have been analyzed in great detail in [27, 28].

The solutions of eqn. (2.7) is given by:

$$
\begin{aligned}
T(R) & =-\frac{2 R\left(1+\frac{N g_{s} l_{s}^{7-p}}{R^{7-p}}\right)}{5-p}+\frac{2(7-p)}{\sqrt{N g_{s} l_{s}^{7-p}}} \frac{R^{\frac{7-p+2}{2}}}{(7-p)^{2}-4} \times \\
& \times{ }_{2} F_{1}\left(\frac{2+(7-p)}{2(7-p)}, \frac{1}{2}, 1+\frac{2+(7-p)}{2(7-p)} ;-\frac{R^{7-p}}{N g_{s} l_{s}^{7-p}}\right),
\end{aligned}
$$

where ${ }_{2} F_{1}$ the hypergeometric function. Note that the above solution is not valid for $p=5$, where the form of the solutions and the asymptotic behave differently [26], and we will also present the solution, for completeness, in the case of D5-brane background later on.

The asymptotic behaviour of $T(R)$ can be found out by using the properties of hypergeometric function ${ }_{2} F_{1}: \lim _{R \rightarrow 0}{ }_{2} F_{1} \rightarrow 1$, and $\lim _{R \rightarrow \infty}{ }_{2} F_{1} \rightarrow 0$. Let's analyze the behaviour of the function $T(R)$ and the tachyon potential $V(T(R))$ asymptotically. As $R \rightarrow 0, T(R) \rightarrow-\infty:$

$$
T(R \rightarrow 0) \simeq-R^{p-6}
$$

And, as $R \rightarrow \infty, T(R) \rightarrow 0$ :

$$
T(R \rightarrow \infty) \simeq R^{\frac{p-7}{2}} .
$$

The above can be understood from the simple fact that $p \leq 4$, so $7-p=+v e{ }^{2}$

\footnotetext{
${ }^{2}$ We are excluding the D5 and D6-brane from the above analysis and for other higher branes the harmonic functions behave differently
} 
The effective potential $V(T)$ in various limits behaves as:

$$
\begin{aligned}
\frac{1}{\tau_{p^{\prime}}} V(T) & \simeq T^{\frac{(7-p)\left(p-p^{\prime}-4\right)}{4(6-p)}}, \quad R \rightarrow 0 \\
& \simeq\left(1+c T^{2}\right)^{\frac{p-p^{\prime}-4}{4}}, \quad R \rightarrow \infty .
\end{aligned}
$$

We would be interested in analyzing the behaviour of the potential very close to the $D p$-brane, namely in the region $(R \rightarrow 0)$. One can see easily that when $p-p^{\prime}=4$, the effective potential is a constant. When $p-p^{\prime}<4, V(T) \rightarrow 0$ so there is a possibility of the formation of a bound state. And finally, when $p-p^{\prime}>4, V(T) \rightarrow \infty$, indicating that that the effective potential is repulsive [28].

Now, we would like to analyze the rolling of the $p^{\prime}$-brane in $D p$-brane background when $p-p^{\prime}<4$ and $R \rightarrow 0$. A classic example would then be the system of $D p-D(p+2)$ branes. We would like to mention that unlike the case of D-NS5 system, where there is no perturbative tachyon between the D-brane and NS5- brane, in the present case there is a tachyon in the open string between $D p$ and $D(p+2)$ branes. The bound state is formed due to the dynamics of the tachyon in the open string stretching between the $D p$ and $D(p+2)$ branes as it essentially stabilises the potential [30. In the present case, however, we analyze the motion of $p$-brane in the background of $(p+2)$-brane where the open string tachyon is switched off throughout the dynamical process. So the 'radial mode' of the brane, which behaves exactly like the tachyon and which is the only other field that has been turned on, would determine the rolling of the probe brane in this background when both come close to each other. Infact, if we start with the system with the perturbative tachyon turned on and try to analyze the dynamics, it would then become a coupled system and the above analysis in terms of the radial mode only is no more valid.

Let's now recalculate some quantities in the Hamiltonian formulation from the effective action (2.5), following the derivation of [5]. The energy momentum tensor $T_{a b}$ computed from the action (2.5) is given by:

$$
T_{a b}=\tau_{p^{\prime}} H_{p}^{\frac{p-p^{\prime}-4}{4}}\left[\frac{H(R) \partial_{a} R \partial_{b} R}{\sqrt{1+H(R) \eta^{c d} \partial_{d} R \partial_{c} R}}-\eta_{a b} \sqrt{1+H(R) \eta^{c d} \partial_{d} R \partial_{c} R}\right]
$$

The solutions to the equations of motion described by the action (2.5) can easily then be computed by working in hamiltonian formalism. The momentum conjugate to $R$ is:

$$
\Pi=\frac{\tau_{p^{\prime}} H_{p}^{\frac{p-p^{\prime}}{4}} \partial_{0} R}{\sqrt{1-H\left(\partial_{0} R\right)^{2}+H(\vec{\nabla} R)^{2}}}
$$


We can construct the Hamiltonian $H$ :

$$
\begin{aligned}
& H=\int d^{p^{\prime}} x\left(\Pi \partial_{0} R-\mathcal{L}\right)=\int d^{p^{\prime}} x \mathcal{H}, \\
& \mathcal{H}=T_{00}=\sqrt{\frac{\Pi^{2}}{H_{p}}+V^{2}(T)} \sqrt{1+H_{p}(\vec{\nabla} R)^{2}},
\end{aligned}
$$

where $V(T)=\tau_{p^{\prime}} H^{\frac{p-p^{\prime}-4}{4}}$. The equation of motion for $R$ is given by:

$$
\partial_{0} R=\frac{\delta H}{\delta \Pi}=\frac{\Pi}{H_{p}} \frac{\sqrt{1+H_{p}(\vec{\nabla} R)^{2}}}{\sqrt{\frac{\Pi^{2}}{H_{p}}+V^{2}(T)}} .
$$

As discussed above, we will be interested in the dynamics of the $D p^{\prime}$-brane in the region where $T \rightarrow-\infty(R \rightarrow 0)$ and $p-p^{\prime}<4$. So one can ignore the potential $V(T)$ (first line of eqn. 2.12) and the rest of the dynamics is governed by the 'tachyon' (that is the Radial mode $R$ ). The hamiltonian and the equations of motion take the following simpler form:

$$
\begin{gathered}
H=\int d^{p^{\prime}} x \frac{|\Pi|}{\sqrt{H_{p}}} \sqrt{1+H(\vec{\nabla} R)^{2}}, \\
\partial_{0} R=\frac{\delta H}{\delta \Pi}=\frac{1}{\sqrt{H_{p}}} \frac{\Pi}{|\Pi|} \sqrt{1+H_{p}(\vec{\nabla} R)^{2}}
\end{gathered}
$$

Form eqn (2.18) we get:

$$
H_{p}\left[\left(\partial_{0} R\right)^{2}-(\vec{\nabla} R)^{2}\right]=1
$$

When translated in terms of the variables $T$, one gets:

$$
\left(\partial_{0} T\right)^{2}-(\vec{\nabla} T)^{2}=1
$$

As explained in [5], the above equations of motions when transformed into the old variable $T$, have the natural explanations of equations governing the motion of nonrotating and non-interacting dust. It has been proved in [3] that the solutions describing the tachyon on a brane-antibrane system near the minimum does not admit any plane-wave solutions. The pressure falls to zero exponentially at very late time as the tachyon field evolves from any spatially homogeneous initial configuration towards the minimum. In the case of D-brane near the NS5-brane, this has also been argued in [26]. So it will be interesting to find out the field theory of the 'tachyon matter' in these set ups. 


\section{Homogeneous Solutions}

In this section, we would like to study some homogeneous solutions to the equations of motion explained in the earlier section. We assume that the fields in the common transverse directions are functions of time only $\left(X^{m}(t)\right)$. If this is the case then there is no inhomogeneity in the initial data and the caustics explained in [31] don't form at finite time. Hence the Born-Infeld analysis is appropriate, and the motion of Dbranes can be analyzed by that. Let's assume then the coordinates in the common transverse directions $X^{m}(m=p+1, \ldots, 9)$, depend only on time. So the induced metric on the worldvolume of $D p^{\prime}$-brane takes the form:

$$
G_{a b}=H_{p}^{-\frac{1}{2}} \eta_{a b}+\delta_{a}^{0} \delta_{b}^{0} \dot{X}^{m} \dot{X}^{m} H_{p}^{\frac{1}{2}}
$$

Now substituting the metric and the dilaton into the action (2.5), we get:

$$
\mathcal{S}_{p^{\prime}}=-\tau_{p^{\prime}} V \int d t H_{p}^{\frac{p-p^{\prime}-4}{4}} \sqrt{1-\dot{X}^{m} \dot{X}^{m} H_{p}}
$$

where $V$ is the volume of the $p^{\prime}$ dimensional space. It is easy to find out the momentum $P_{n}$ by varying the above Lagrangian with respect to $\dot{X}^{n}$ :

$$
P_{n}=\frac{\delta \mathcal{L}}{\delta \dot{X}^{n}}=\frac{\tau_{p^{\prime}} V H_{p}^{\frac{p-p^{\prime}}{4}} \dot{X}_{n}}{\sqrt{1-\dot{X}^{m} \dot{X}^{m} H_{p}}}
$$

and the total energy is given by:

$$
E=P_{n} \dot{X}^{n}-\mathcal{L}=\frac{\tau_{p^{\prime}} V H_{p}^{\frac{p-p^{\prime}}{4}}}{H_{p} \sqrt{1-\dot{X}^{m} \dot{X}^{m} H_{p}}}
$$

Another quantity of interest is to calculate the Stress tensors $T_{\mu \nu}$ :

$$
\begin{aligned}
T_{00} & =\frac{\tau_{p^{\prime}} H_{p}^{\frac{p-p^{\prime}}{4}}}{H_{p} \sqrt{1-\dot{X}^{m} \dot{X}^{m} H_{p}}}, \\
T_{i j} & =-\tau_{p^{\prime}} \delta_{i j} H^{\frac{p-p^{\prime}-4}{4}} \sqrt{1-H_{p} \dot{X}^{m} \dot{X}^{m}} \\
T_{0 i} & =0 .
\end{aligned}
$$

From the above, it is not difficult to see that the pressure goes to zero at very late times, so the system behaves like a pressure-less gas. It would be interesting to work 
out the case when there is a background NS-NS flux and/or a worldvolume gauge field, and examine the behaviour of the pressure $\left(\propto T_{i j}\right)$ and see how the background flux is playing a role in the motion of the brane and the eventually the time dynamics of the 'tachyon'. Similar issues regarding the D-brane decay in the presence of a background electric field from the two-dimensional conformal field theory point of view has been addressed in 10 and the final decay product has been shown to be a fundamental string and a "tachyon matter". By using the T-duality transformation on the relevant boundary state, such decay process has also been studied in 32, 33. It would be nice to repeat the analysis of this paper in the presence of an electric (magnetic) field and study the time evolution process.

\section{D-brane dynamics in D5-brane background}

In this section, we would like to analyze the dynamics of D-branes in the background of $N$ coincident D5-branes where all of them lie parallel to each other and the worldvolume directions of the probe brane falls into the directions parallel to the D5-branes. The metric, dilaton and the R-R flux of a system of $N$ coincident D5-branes is given by:

$$
\begin{aligned}
g_{\alpha \beta} & =H^{-\frac{1}{2}} \eta_{\alpha \beta}, \quad g_{m n}=H^{\frac{1}{2}} \delta_{m n}, \quad(\alpha, \beta=0, . ., 5 ; m, n=6, . ., 9) \\
e^{2 \phi} & =H^{-1}, \quad F_{m n p}=-\epsilon_{m n p}^{r} \partial_{r} H .
\end{aligned}
$$

where $H=1+\frac{N g_{s} l_{s}^{2}}{r^{2}}$ is the Harmonic function in the four transverse directions of the D5-branes. The DBI action can be read off from eqn. (2.5). The tachyon field and the tachyon effective potential is given by:

$$
\frac{d T}{d R}=\sqrt{H(R)}=\sqrt{1+\frac{N g_{s} l_{s}^{2}}{R^{2}}},
$$

and

$$
V(T)=\frac{\tau_{p^{\prime}}}{[H(R(T))]^{\frac{p^{\prime}-1}{4}}} .
$$

In this case however, the $T(R)$ in a monotonically increasing function [26]:

$$
T(R)=\sqrt{N g_{s} l_{s}^{2}+R^{2}}+\frac{1}{2} \sqrt{N g_{s}} l_{s} \ln \frac{\sqrt{N g_{s} l_{s}^{2}+R^{2}}-\sqrt{N g_{s}} l_{s}}{\sqrt{N g_{s} l_{s}^{2}+R^{2}}+\sqrt{N g_{s}} l_{s}} .
$$


As $R \rightarrow 0, T(R) \rightarrow-\infty:$

$$
T(R \rightarrow 0) \simeq \ln R .
$$

As $R \rightarrow \infty, T(R) \rightarrow \infty:$

$$
T(R \rightarrow \infty) \simeq R
$$

The effective potential in these two asymptotic regions is given by:

$$
\begin{aligned}
\frac{1}{\tau_{p^{\prime}}} V(T) & \simeq[\exp T]^{\frac{p^{\prime}-1}{2}}, T \rightarrow-\infty \\
& \simeq\left[1-\frac{\left(p^{\prime}-1\right)}{4} \frac{N g_{s} l_{s}^{2}}{T^{2}}\right], \quad T \rightarrow \infty .
\end{aligned}
$$

One can see that for $p^{\prime}=1$, the potential goes to a constant in both cases and the force goes to zero. In the limit $T \rightarrow-\infty$, which correspond to $R \rightarrow 0$, one finds that the potential goes to zero exponentially. This is the expected late time behaviour for the tachyon potential in the rolling tachyon solutions. One can also compute the energy momentum tensors and can show that the pressure falls off exponentially at a very late time. We would like to point out that the above analysis is valid for the region $N g_{s} \gg 1$ where the description in terms of closed string background makes sense. The systematics of the D-brane motion in this D5-brane background can be analyzed by following [26. In this case, it is much simpler to show the absence of plane wave solutions near the minimum of the potential $V(T)$. It is also somewhat straightforward to show the possible trajectories of the probe brane in the directions transverse to the D5-brane, after passing to the polar coordinates, following the derivations of [26]. We however skip those details here.

\section{Conclusion}

In this paper, we have studied the D-brane dynamics in the background that is generated by a stack of static branes from the point of view of the DBI action. The study of real time dynamics of the D-brane provides an example of time dependent process in string theory. We have shown that when there is no WZ interaction between the probe and the background branes, one can map this problem into a similar problem of tachyon condensation and study the time evolution process. We discuss the classical Hamiltonian formulation and study some homogeneous solutions to the equations of motion. We study the interaction potential and show that the pressure goes to zero at very late times. Finally we remark on D-brane dynamics in the presence of D5-

brane which shows that the pressure goes to zero at late times exponentially. There 
are further questions which needs to be addressed. Though the analysis of brane dynamics can be mapped, in these simple cases, to a problem of real time tachyon dynamics of the open string theory, it is not clear how all the effects like the gauge fields and the other R-R fields could be incorporated in these studies. For example: it has been discussed in [28, that a possible WZ interaction could arise between the background and the probe brane when the dimensionality between the two differs by six: $\left(p+p^{\prime}=6\right)$. The D-brane trajectory has been shown, in that context, to be equivalent to that of a non-relativistic dyon in a magnetic monopole background. So it would be interesting to repeat the above analysis to notice the changes in the energy momentum tensors and study the time evolution process. One way, however, is to construct boundary state of the systems and study the properties of stress tensors to analyze the behaviour of 'tachyon matter'. Another interesting aspect would be to discuss the D-brane dynamics in more detail in the presence of various other non-perturbative objects and learn more about the time dependent classical solutions of string theory. Of course an interesting exercise would be lift the discussion of the DBI to that of the full conformal field theory analysis. In that picture, the properties of the classical time dependent solutions would correspond to perturbing the original D-branes by an exactly marginal deformation and study its effects. More recently the boundary states of rolling D-branes in NS5-brane background are constructed in 34. So it will be interesting to construct, if possible, more generalized boundary states of Rolling D-branes in the presence of various flux. In the brane picture it would be useful to show how these problems can be analyzed from more generalized set ups and the possible connections with the cosmology (a related problem has been addressed recently in 35]). We hope to return to some of these issues in near future.

Acknowledgment: I am grateful to A. Sen for suggestions on the manuscript and for various useful discussions. I would like to thank M. Bianchi, J. David, J. F. Morales, R. R. Nayak and especially A. Sagnotti for numerous interesting discussions. This work was supported in part by I.N.F.N., by the E.C. RTN programs HPRNCT-2000-00122 and HPRN-CT-2000-00148, by the INTAS contract 99-1-590, by the MURST-COFIN contract 2001-025492 and by the NATO contract PST.CLG.978785.

\section{References}

[1] A. Sen, "Rolling Tachyon", JHEP 0204, 048 (2002) arXiv:hep-th/0203211.

[2] A. Sen, "Tachyon Matter", JHEP 0207, 065 (2002) arXiv:hep-th/0203265.

[3] A. Sen, "Field Theory of Tachyon Matter", Mod.Phys.Lett. A17 1797 (2002), arXiv:hep-th/0204143 
[4] A. Sen, "Time evolution in open string theory," JHEP 0210003 (2002), arXiv:hep-th/0207105.

[5] A. Sen, "Time and Tachyon", Int.J.Mod.Phys. A18 4869 (2003), arXiv:hep-th/0209122

[6] M. Gutperle, and A. Strominger, "Spacelike Branes", JHEP 0204, 018 (2002) arXiv:hep-th/0202210.

[7] S. Sugimoto, and S. Terashima, "Tachyon Matter in Boundary String Field Theory" JHEP 0207, 025 (2002) arXiv:hep-th/0205085.

[8] J. A. Minahan, "Rolling the tachyon in super BSFT," JHEP 0207, 030 (2002) arXiv:hep-th/0205098.

[9] K. Hashimoto, "Dynamical decay of brane-antibrane and dielectric brane," JHEP 0207, 035 (2002) arXiv:hep-th/0204203.

[10] P. Mukhopadhyay, and A. Sen, "Decay of Unstable D-branes with Electric Field", JHEP 0211047 (2002), [arXiv: hep-th/0208142].

[11] A. Sen, "Supersymmetric World-volume Action for Non-BPS D-branes" JHEP 9910008 (1999), arXiv:hep-th/9909062

[12] M. R. Garousi, "Tachyon couplings on non-BPS D-branes and Dirac-Born-Infeld action", Nucl. Phys. B584 284 (2000) arXiv:hep-th/0003122

[13] E.A. Bergshoeff, M. de Roo, T.C. de Wit, E. Eyras, and S. Panda, "T-duality and Actions for Non-BPS D-branes", JHEP 0005009 (2000) arXiv:hep-th/0003221.

[14] J. Kluson, "Proposal for non-BPS D-brane action", Phys.Rev. D62 126003 (2000) arXiv:hep-th/0004106.

[15] A. Sen, "Open-Closed Duality at Tree Level", Phys. Rev. Lett. 91181601 (2003), arXiv:hep-th/0306137

[16] A. Sen, "Open-Closed Duality: Lessons from Matrix Model" Mod.Phys.Lett. A19 841 (2004), arXiv:hep-th/0308068

[17] G. W. Gibbons, "Cosmological Evolution of the Rolling Tachyon", Phys.Lett. B537 1 (2002), arXiv:hep-th/0204008.

[18] L. Kofman, and A. Linde, "Problems with Tachyon Inflation", JHEP 0207004 (2002), arXiv:hep-th/0205121 
[19] M. Fairbairn, and M. H.G. Tytgat, "Inflation from a Tachyon Fluid ?" Phys. Lett. B546 1 (2002), [arXiv: hep-th/0204070].

[20] S. Mukohyama, Brane cosmology driven by the rolling tachyon Phys.Rev. D66 024009 (2002), arXiv:hep-th/0204084.

[21] T.Padmanabhan, "Accelerated expansion of the universe driven by tachyonic matter", Phys. Rev. D66 021301 (2002) arXiv:hep-th/0204150

[22] C.P. Burgess, M. Majumdar, D. Nolte, F. Quevedo, G. Rajesh, and R.-J. Zhang, "The Inflationary Brane-Antibrane Universe", JHEP 0107047 (2001), arXiv hep-th/0105204.

[23] D. Choudhury, D. Ghoshal, D. P. Jatkar, and S. Panda, "On the Cosmological Relevance of the Tachyon", Phys.Lett. B544 231 (2002), arXiv:hep-th/0204204.

[24] A. Sen, "Remarks on Tachyon Driven Cosmology" arXiv:hep-th/0312153.

[25] M. Sami, P. Chingangbam, and T. Qureshi, "Aspects of Tachyonic Inflation with Exponential Potential", Phys. Rev. D66 043530 (2002), arXiv:hep-th/0205179.

M. R. Garousi, M. Sami, and S. Tsujikawa, "Cosmology from Rolling Massive Scalar Field on the anti-D3 Brane of de Sitter Vacua", arXiv:hep-th/0402075.

[26] D. Kutasov, "D-Brane Dynamics Near NS5-Branes", [arXiv: hep-th/0405058.

[27] C. P. Burgess, P. Martineau, F. Quevedo, and R. Rabadan, "Branonium", JHEP 0306, 037 (2003) [arXiv: hep-th/0303170

[28] C. P. Burgess, N. E. Grandi, F. Quevedo, and R. Rabadan, "D-Brane Chemistry", JHEP 0401067 (2004), [arXiv: hep-th/0310010

[29] R. C. Myers, "Dielectric Branes", JHEP 9912022 (1999), [arXiv: hep-th/9910053.

[30] E. Gava, K. S. Narain, and M. H. Sarmadi, "On the Bound States of p- and (p+2)-Branes", Nucl. Phys. B504 214 (1997), [arXiv: hep-th/9704006].

[31] G. N. Felder, L. Kofman, and A. Starobinsky, "Caustics in Tachyon Matter and Other Born-Infeld Scalars", JHEP 0209026 (2002), arXiv:hep-th/0208019

G. N. Felder, and L. Kofman, Inhomogeneous Fragmentation of the Rolling Tachyon, arXiv:hep-th/0403073,

[32] S.-J. Rey, and S. Sugimoto, "Rolling Tachyon with Electric and Magnetic Fields T-duality approach -", Phys. Rev. D67 086008 (2003), arXiv:hep-th/0301049. 
[33] S.-J. Rey, and S. Sugimoto, "Rolling of Modulated Tachyon with Gauge Flux and Emergent Fundamental String", Phys. Rev. D68 026003 (2003), arXiv:hep-th/0303133.

[34] Y. Nakayama, Y. Sugawara, and H. Takayanagi, "Boundary States for the Rolling D-branes", arXiv:hep-th/0406173.

[35] H. Yavartanoo, "Cosmological Solution from D-brane motion in NS5-Branes background", arXiv:hep-th/0407079. 